\title{
Application of thermography for painting underdrawing detection
}

by M. Kałuża, M. Kopeć, R. Olbrycht

Lodz Univ. of Technology, 90-924, Wolczanska Str., Lodz, Poland, marcin.kaluza@p.lodz.pl

\section{Abstract}

The aim of this paper is to present the application of infrared thermography for painting underdrawing detection. Working with two paintings on canvas prepared especially for the purpose of this work, the authors used a cooled InSb thermal camera for the detection of the unknown underdrawings and compared them with the images obtained using a standard CCD camera equipped with a filter blocking visible light. The traditional visualization in reflected infrared light was also compared to the approach based on infrared radiation transmission.

\section{Introduction}

One of the techniques used by painters consist in sketching the planned composition of the artwork directly on the ground layer, and to later follow it more or less precisely when brushing, following the personal preferences of the author. Some form of sketching is also used by counterfeiters, who on many occasions, when duplicating the original painting, are creating additional grid marks on the surface of the forged canvas to help them replicate the original artwork. Thus, the hidden sketch is a source of valuable information for painting technique investigation, restoration, forgery detection and cultural heritage preservation.

The painting has a multilayer structure consisting of the canvas as the base, covered with the ground layer, followed by the underdrawing, paint, and finally the protective varnish layer (fig. 1). Each of those layers has different spectral properties. In [1-2] the author proposed a non-destructive technique called infrared reflectography in which the painting was illuminated and the reflected image was observed using a vidicon television system sensitive in the NIR and SWIR band up to $2 \mu \mathrm{m}$. The system was equipped with a band-pass filter blocking the visible light. Because in the mentioned IR band pigments layers of the artwork have distinctively different properties, comparing to visible band, and the carbon based underdrawing layer is strongly absorbing infrared, it was possible to detect and visualize the underdrawing. The same technique was later used by many researchers, replacing the vidicon system by NIR CCD cameras (sensitive up to $1.1 \mu \mathrm{m}$ ) or lately SWIR InGaAs systems (sensitive up to $1.7 \mu \mathrm{m}$ ) [3-5]. As it was demonstrated in [6-7], a multispectral approach for non-destructive investigation of paintings on different substrates allows to gather much more information about them, comparing to a single band approach.

Based on above, the authors of this article investigated the possibility of using a single thermographic camera as a tool for multiband investigation of paintings on canvas.

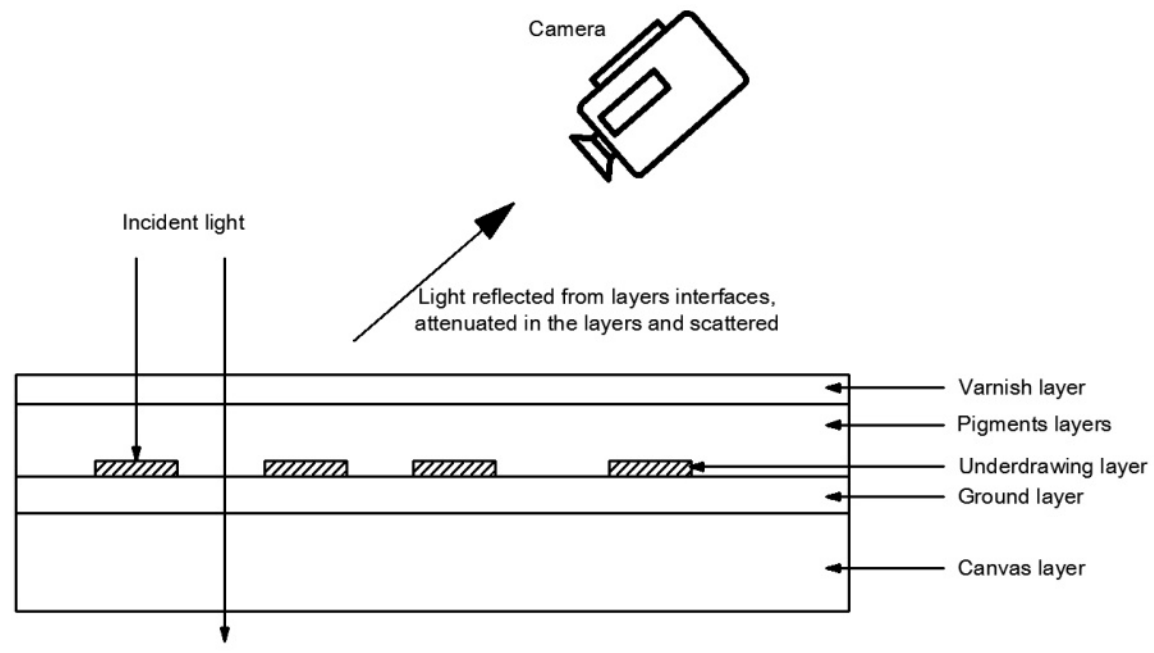

Fig. 1. Painting multilayer structure - reflectography concept 


\subsection{1/qirt.2016.050}

\section{Measurement setup and results}

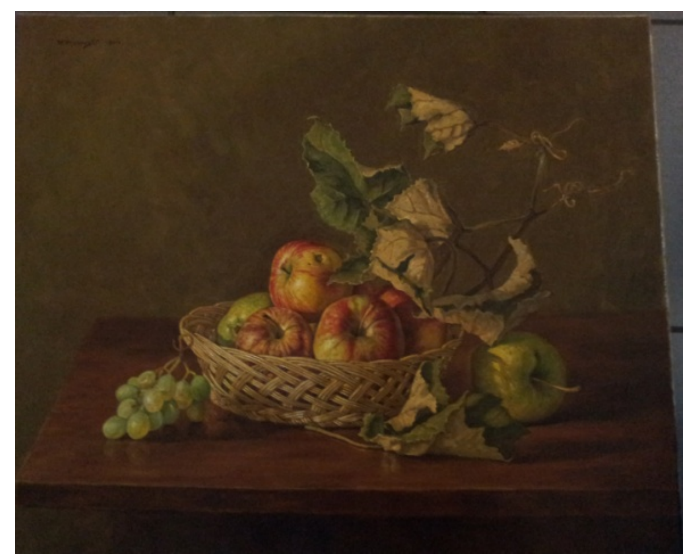

Fig. 2. Painting used for investigation

For the purpose of the investigation, two different paintings on canvas were prepared with unknown underdrawings hidden beneath the paint layer. One of them is shown in fig. 2. The artwork was first investigated using a Bassler acA640$100 \mathrm{gm}$ CCD camera offering a 659 × 494 pixels resolution. A series of images of the painting were acquired using a halogen lamp as light source. Four different setups were used and compared, i.e. the camera with an IR filter blocking visible light and the camera without any filter, light reflected from the painting and light transmitted through the canvas (backlighting). The results obtained for the right lower part of the painting from fig. 2 are shown below.

a)

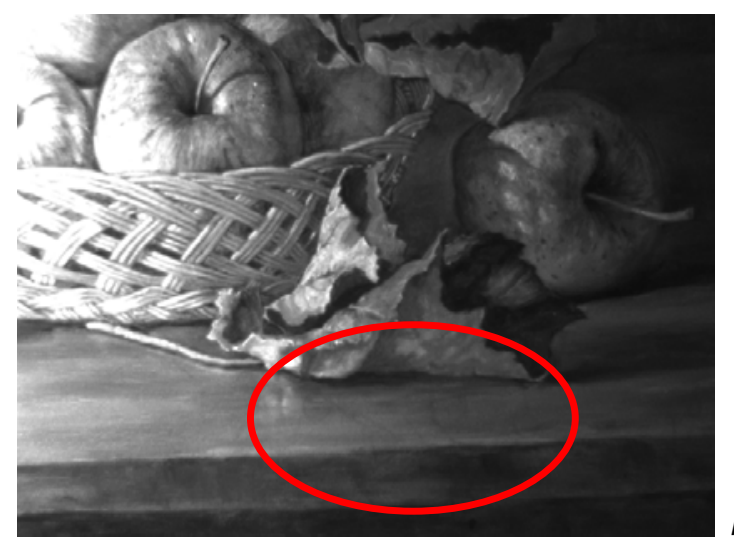

b)
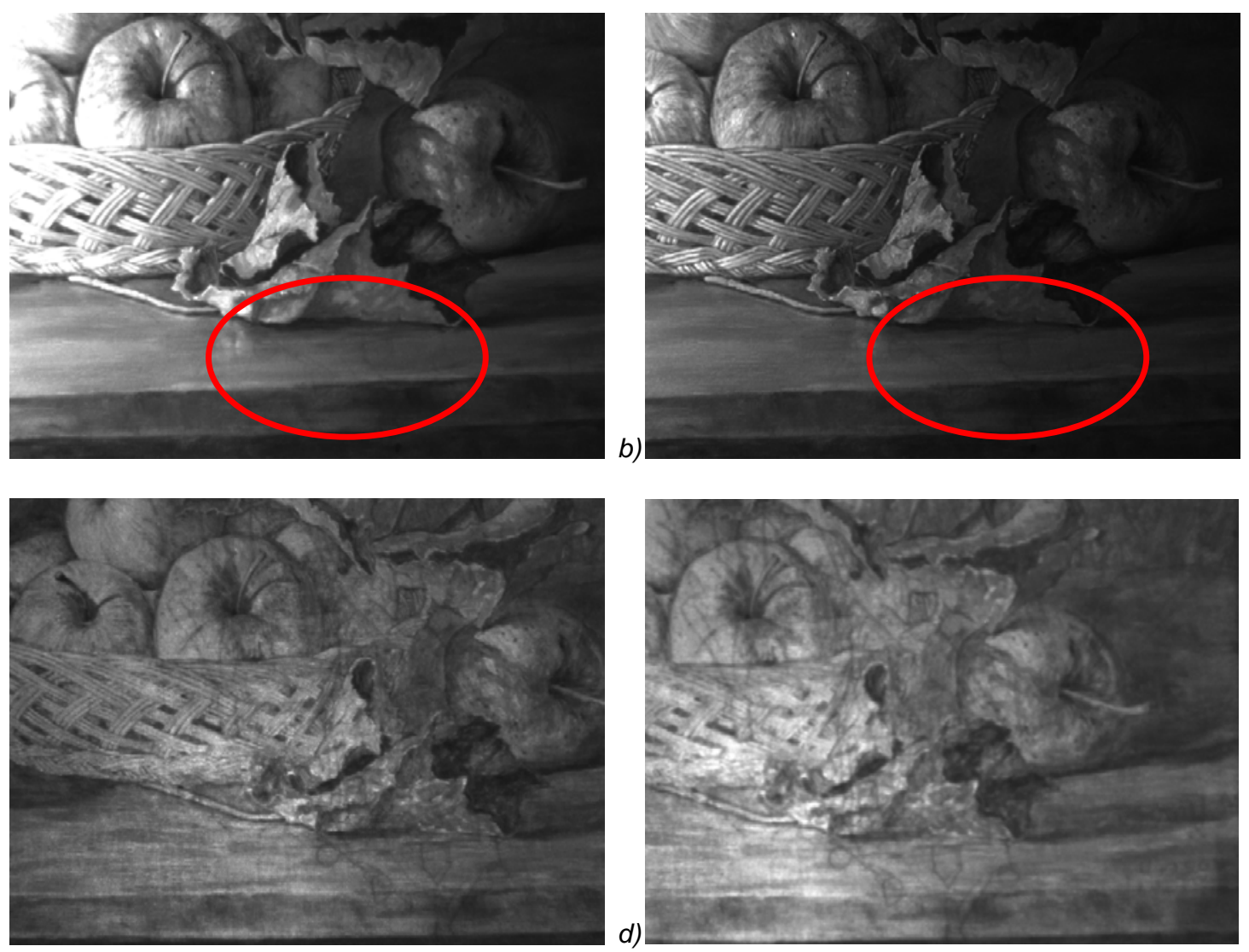

Fig. 3. Painting images obtained using a Bassler acA640-100gm CCD camera:

a) camera without filter, reflected light; b) camera with an IR filter, reflected light;

c) camera without filter, light transmitted through the canvas; d) camera with an IR filter, light transmitted through the canvas 


\subsection{1/qirt.2016.050}

Working with the camera without any filter it was possible to distinguish the outline of a building roof in the area were dark brown paint was used (fig. 3a). Adding to the camera an IR filter blocking visible light reflected from the painting layer slightly increased the contrast of the discovered underdrawing elements (fig. 3b). In general, no underdrawing was detected in the areas where light colours were applied. Much better results were obtained when the halogen light source was placed behind the canvas (fig. 3c), allowing to detect much more elements of the underdrawing than when using standard reflectography approach. Again in this case, adding an IR filter increased the contrast and visibility of the underdrawing, which can be seen in the area containing the basket with apples (fig. 3d). Also in this case areas covered with light/creamy colors remained to some extent opaque. If any sketch was present, it remained invisible. When using the backlight configuration, the power setting of the lamp had an influence on the underdrawing visibility. The stronger was the illumination, the better was the sketch contrast.

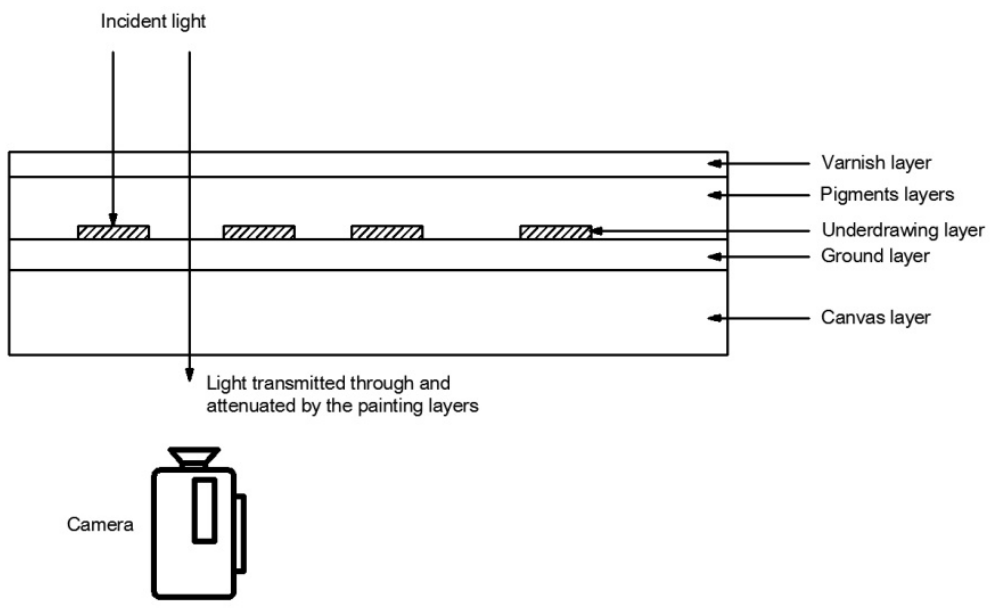

Fig. 4. Painting multilayer structure - back-illumination approach

In the traditional reflectography approach, the camera receives the light reflected from the layers interfaces, attenuated in the layers and scattered. If pigments characterized by a strong reflection in the band of interest are present, light reflected from their surface will mask the underdrawing. If for the same painting and equipment, the back-illumination configuration will be used (fig. 4), the light in the sketch areas will be strongly attenuated, whereas in the remaining parts of the artwork more light will be transmitted through - assuming a strong enough illumination - providing contrast and possibility of underdrawing visualisation.

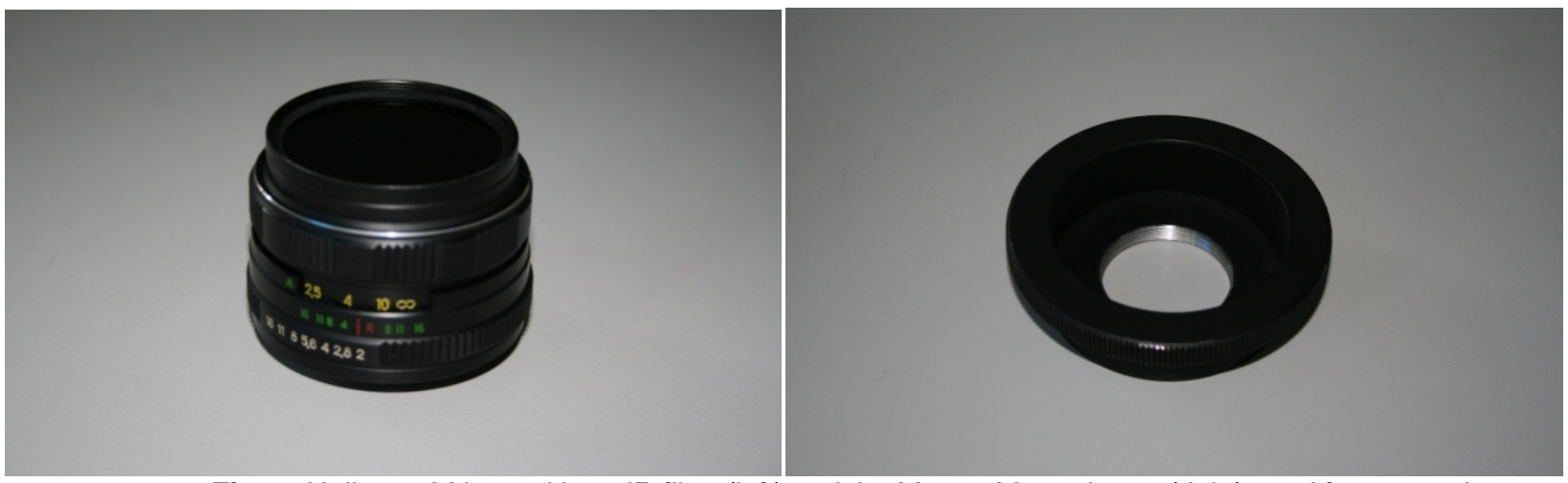

Fig. 5. Helios $44 M$ lens with an IR filter (left) and the M42 to M80 adapter (right) used for research

In the next step, the paintings were investigated using a Cedip Titanium MWIR camera with an InSb 640x512 pixels cooled detector matrix. When using the standard $50 \mathrm{~mm}$ lens with a M80 mount, this camera operates in the 3,6$4,7 \mu \mathrm{m}$ band limited by the filtering coating of the lens. Because of the wideband characteristic of the InSb detector matrix (approx. 1-5 $\mu \mathrm{m}$ ), a M42 to M80 adapter was manufactured allowing to replace the original lens with a lens coming from a $35 \mathrm{~mm}$ film camera. To avoid any IR blocking coatings, a Helios 44M lens was chosen. To confirm that choice, its spectral transmission characteristics was measured, giving a $80 \%$ light transmission up to $2.2 \mu \mathrm{m}$ and a cut-off wavelength of around $2.7 \mu \mathrm{m}$.

Using the setup described above, the paintings were imaged, illuminated from the front (reflectography) or backilluminated with the same halogen lamp as during the first experiments. The measurements were repeated using a $720 \mathrm{~nm}, 760 \mathrm{~nm}, 850 \mathrm{~nm}$ and $950 \mathrm{~nm}$ IR filter mounted on the Helios lens. Some of the results obtained for the painting from fig. 2 are shown in fig. 6 below. 


\subsection{1/qirt.2016.050}
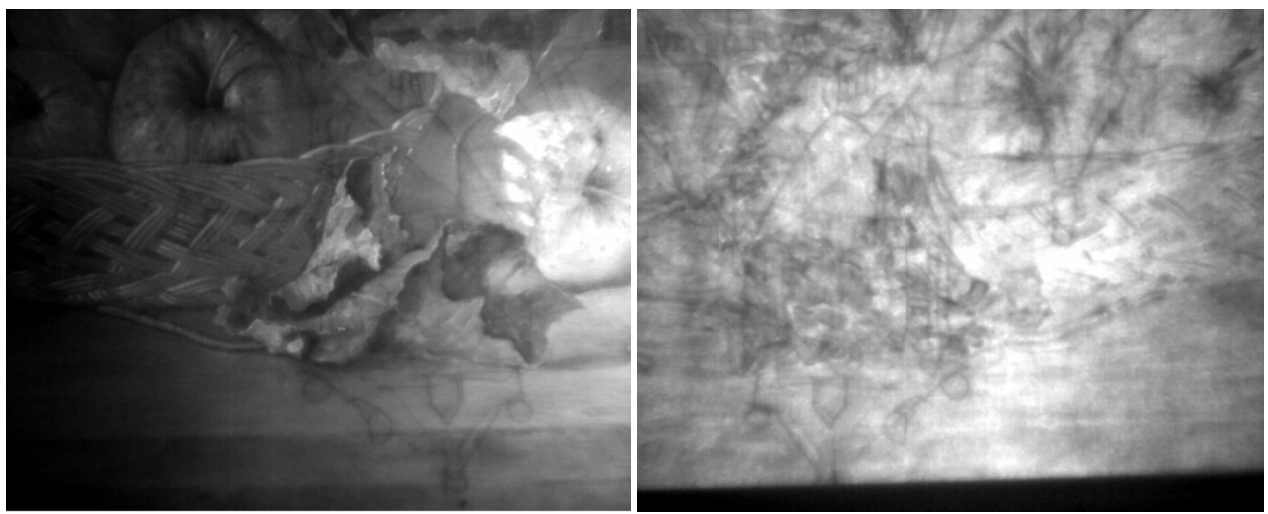

Fig. 6. Image of the painting seen by the thermographic camera using a Helios 44M lens: reflected illumination (left), back-illumination (right)

When comparing the results from fig. 3 and fig. 6 , both in the reflected and backlighted configuration, the thermographic camera gave better results than the CCD one. The choice of the IR filter had practically no impact on the results. This can be explained by the spectral sensitivity curve of the InSb focal plane array (FPA) used in the thermographic camera, which operates approximately above $1 \mu \mathrm{m}$ wavelength, thus naturally acting as a high-pass filter. This provided a better contrast for lighter pigments and, as described above, better results when comparing with the Bassler CCD camera.

Another element that had to be taken into account when comparing the thermographic vs. CCD camera was the light source. As stated above, the CCD camera is sensitive approximately up to $1.1 \mu \mathrm{m}$ whereas the InSb FPA operates in the 1-5 $\mu \mathrm{m}$ band. A typical halogen lamp, as the one used for the experiments, has an emission spectrum of up to $1.2 \mu \mathrm{m}$ wavelength, which matches the CCD array sensitivity band but should be considered as ill-adapted to the InSb FPA, not allowing to use its full potential. Given above, the last described experiment was repeated, replacing the halogen lamp with a quartz tungsten lamp, which is a source of light offering a much wider radiation band, well beyond the $1-2.7 \mu \mathrm{m}$ band of interest limited for short wavelengths by the InSb FPA, and the Helios lens for long wavelengths. Some of the results obtained for the painting from fig. 2 are shown in fig. 7 below.
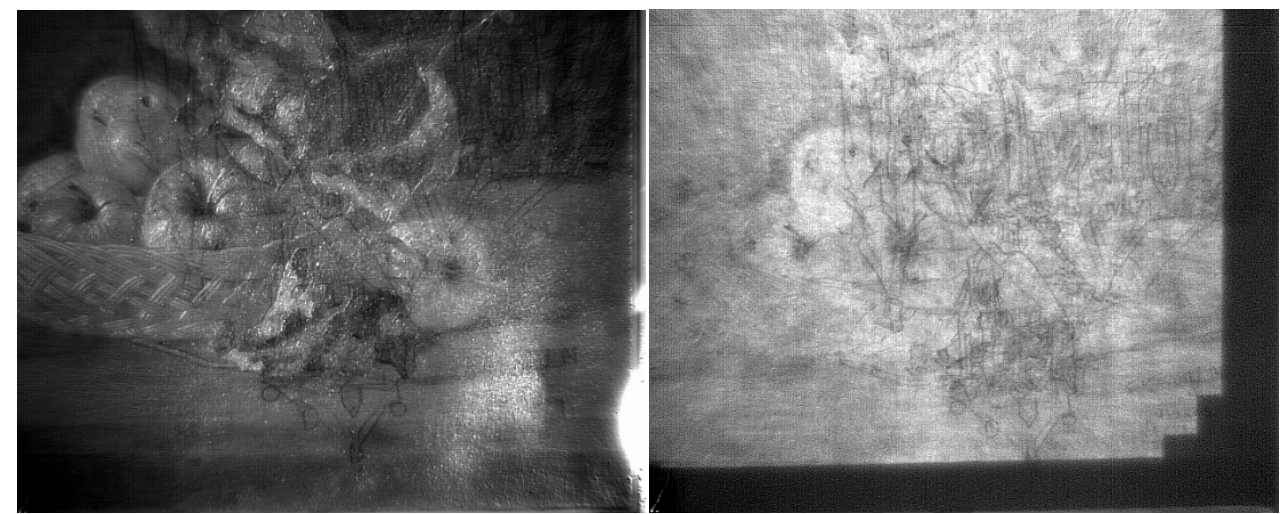

Fig. 7. Image of the painting seen by the thermographic camera using a Helios $44 M$ lens and a quartz tungsten lamp: reflected illumination (left), back-illumination (right)

As can be seen on the pictures above, the emission spectrum of the quartz tungsten light source was better adapted and provided better results than the halogen lamp, which is clearly visible when comparing the figures presented so far in the apples basket area of the painting.

When comparing the obtained thermograms, in all cases the lines of the underdrawing gave an impression of slight fuzziness and lack of sharpness. It was suspected that this problem was related to the limited resolution of the camera FPA and the fact, that to obtain a sharp image of the painting it was necessary to place the camera at least $2 \mathrm{~m}$ from the artwork when using the Helios lens. To verify that hypothesis, a measurement of the slit response function (SRF) of the Cedip camera with the Helios lens was performed using an adjustable slit and a blackbody as radiation source. The measurements showed that a slit opening of at least $7 \mathrm{~mm}$ was required, thus confirming the hypothesis of impact of the camera IFOV limitation on the sharpness of underdrawing imaging. To solve that problem a set of macrophotography dedicated M42 extension rings was used (fig. 8). The rings were inserted between the M42 to M80 adapter and the lens. Some of the results obtained for the painting from fig. 2 are shown in fig. 9 below. 


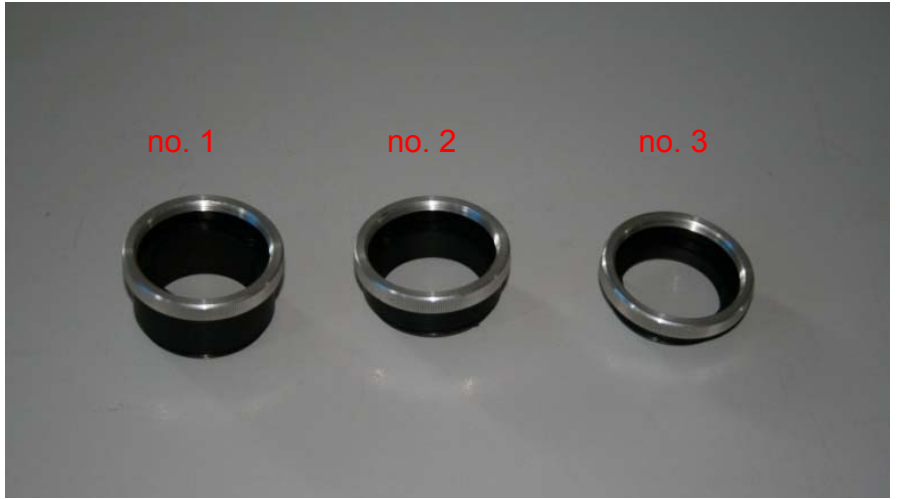

Fig. 8. Macrophotography M42 extension rings used for the experiments

a)

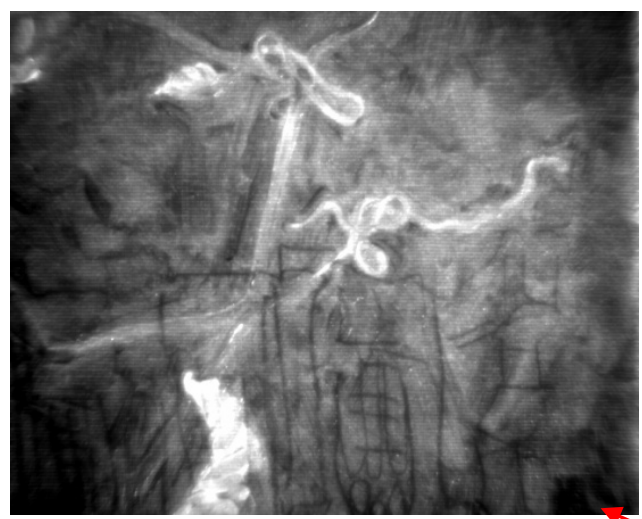

b)
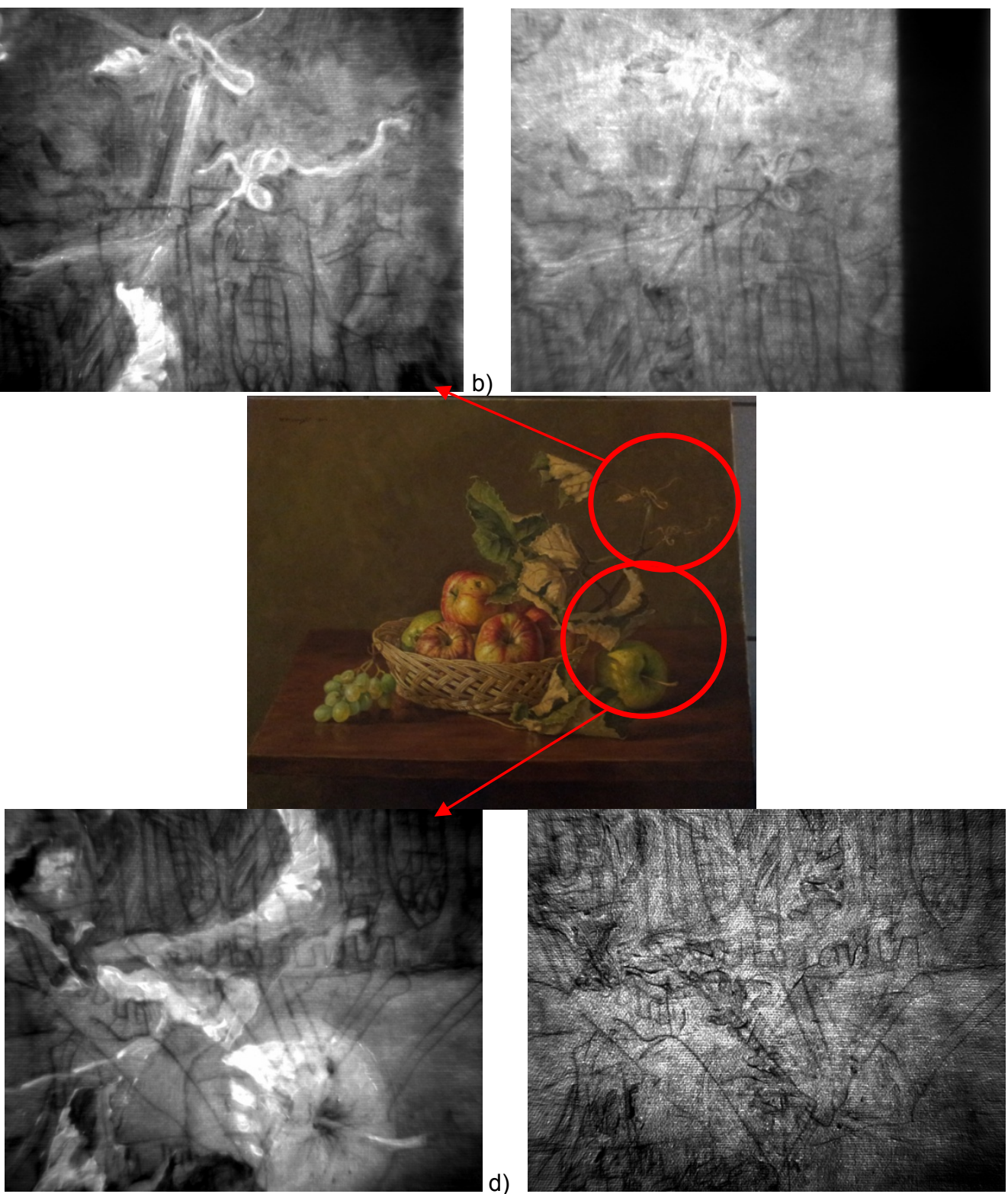

c)

Fig. 9. Image of the painting seen by the thermographic camera using a Helios $44 M$ lens and the extension ring no. 3: reflected illumination (left), back-illumination (right) 


\subsection{1/qirt.2016.050}

The use of an extension ring allowed to reduce the minimum camera focusing distance and to increase the IFOV of the system. As can be seen on the pictures above, this time the underdrawing in the images obtained using reflected illumination is sharper and more pronounced than when using back-illumination. In figure 9c one can also clearly see, that in the parts of the painting, where white/creamy pigments were used, the infrared radiation reflection is strong enough to hide the sketch or make it barely visible. In those areas the backlight approach shows its advantage over the reflected one. If the used backlight is strong enough, the internal structure of the canvas becomes visible (fig. $9 \mathrm{~d})$, allowing to identify any damages or inhomogeneities.

\section{Conclusions}

Using a Cedip Titanium MWIR thermographic camera with a Helios 44M lens, two paintings on canvas were imaged. The setup allowed to visualize the underdrawings normally hidden under the pigments layer. The comparison of the reflected light vs. transmitted light observation showed, that in the case of light pigments, taking into account strong reflections in the near-infrared band, the transmitted light approach gave higher contrast and allowed to identify parts of the underdrawings that were not fully visible in the reflected light configuration. To be able to correctly visualize the sketch hidden under the pigments layers and to be able to use the full potential of the thermal camera, an appropriate, broadband light source is needed, an adequate IR filter mounted on the lens and an adequate IFOV of the imaging system.

\section{REFERENCES}

[1] J.R.J .Van Asperen de Boer, Infrared reflectography: A method for the examination of paintings. Applied Optics, 1968, 7 (9), pp. 1711-1714.

[2] J.R.J .Van Asperen de Boer, Reflectography of Paintings Using an Infrared Vidicon Television System, Studies in Conservation, vol. 14, no. 3, Aug. 1969, pp. 96-118.

[3] J.K. Delaney et. al, Visible and infrared reflectance imaging spectroscopy of paintings: Pigment mapping and improved infrared reflectography. Proc. SPIE 7391, 2009, pp. 739103.1-8.

[4] C. M. Falco, Invited Article: High resolution digital camera for infrared reflectography, REVIEW OF SCIENTIFIC INSTRUMENTS 80, 071301, 2009.

[5] COSENTINO, Panoramic Infrared Reflectography Technical Recommendations, INTERNATIONAL JOURNAL OF CONSERVATION SCIENCE, vol. 5, issue 1, January-March 2014, pp. 51-60.

[6] Wiecek, M. Poksinska, A. Wyrwa, Thermovision investigation of frescos in Cistercian monastery in Lad (Poland), 9th International Conference on Quantitative InfraRed Thermography, July 2-5, 2008.

[7] Daffara, R. Fontana, Multispectral Infrared Reflectography to Differentiate Features in Paintings MICROSCOPY AND MICROANALYSIS, APRIL 2011, pp. 691-695. 